INVESTIGACIONES

\title{
La educomunicación de Don Bosco y la formación de universitarios como buenos ciudadanos
}

\author{
Educommunication of Don Bosco and the formation of university as good citizens
}

A educomunicação de Don Bosco e a formação da universidade como bons cidadãos

\author{
César Andrade-Martinez ${ }^{a}$ \\ ${ }^{a}$ Universidad Politécnica Salesiana, Ecuador. \\ candrade@ups.edu.ec
}

\section{RESUMEN}

Hablar de la Educomunicación de Don Bosco es analizar un tema de gran trascendencia e interés internacional en el campo de la educación y la comunicación, no solo implica conocer sobre el campo de estudio de las mencionadas disciplinas y su contextualización al unirse interdisciplinarmente, su objetivo es conocer la metodología de enseñanza llamado Sistema Preventivo, utilizado por Don Bosco para educar a jóvenes a quienes formó como buenos ciudadanos, enseñando con el amor, la razón y la amabilidad, procedimientos pedagógicos que han servido hasta nuestros tiempos como técnicas de enseñanza. La presente investigación aborda, el concepto y origen de la educomunicación, su desarrollo y expansión por Hispanoamérica, Estados Unidos y Europa. Se expone el método preventivo de Don Bosco, su incidencia positiva en la formación de jóvenes en Italia y su réplica y aplicabilidad por diferentes países, así como en Ecuador donde la comunidad Salesiana lo ha utilizado durante los procesos educativos de varias generaciones. La metodología aplicada fue la descriptiva explicativa de tipo cualitativo, la misma que se expone gracias a un complejo estudio reflexivo inductivo, deductivo y de profundización de la información, basada en la revisión bibliográfica y teórica procedente de estudios anteriores. Los resultados obtenidos, proponen las opciones y los métodos de enseñanza implícitos en el Sistema Preventivo de Don Bosco, investigación que puede ser aplicada por docentes durante la formación de estudiantes universitarios.

Palabras claves: Educomunicación, Sistema Preventivo, Educación, Comunicación, Buenos ciudadanos.

\begin{abstract}
Talk about Don Bosco's educommunication is to analyze a topic of great importance and interest in the field of education and communication, it not only implies knowing about the field of study of the mentioned disciplines and their contextualization by interdisciplinary joining, its objective is to know the teaching methodology called the Preventive System used by Don Bosco to educate young people whom he trained as good citizens, teaching with love, reason and kindness, pedagogical procedures that have served as teaching techniques up to our times. This research covers the concept and origin of educommunication, its development and expansion throughout Latin America, the United States and Europe. Don Bosco's preventive method is exposed, its positive impact on the formation of young people in Italy and its replication and applicability in different countries, as well as in Ecuador where the Salesian community has used it during the educational processes of several generations. The applied methodology was the descriptive explanatory qualitative type, the same one that is exposed thanks to a complete inductive reflective study and in-depth information, based on the bibliographic and theoretical review from previous studies. The results obtained propose the options and the teaching methods implicit in the Don Bosco preventive system, research that can be applied by teachers during the training of university students.
\end{abstract}

Key words: Educommunication, Preventive System, Education, Communication, Good citizens. 


\section{RESUMO}

Falar da Educomunicação de Dom Bosco é analisar um tema de grande importância e interesse internacional no campo da educação e da comunicação, não implica apenas conhecer o campo de estudo das referidas disciplinas e a sua contextualização por meio da interdisciplinaridade, tem como objetivo Conhecer a metodologia de ensino chamada Sistema Preventivo, utilizada por Dom Bosco para educar os jovens que formou como bons cidadãos, ensinando com amor, razão e bondade, procedimentos pedagógicos que serviram de técnica pedagógica até os nossos dias. Esta pesquisa aborda o conceito e a origem da educomunicação, seu desenvolvimento e expansão na América Latina, Estados Unidos e Europa. É exposto o método preventivo de Dom Bosco, seu impacto positivo na formação dos jovens na Itália e sua replicação e aplicabilidade em diversos países, assim como no Equador, onde a comunidade salesiana o utilizou durante os processos educativos de várias gerações. A metodologia aplicada foi do tipo descritiva explicativa qualitativa, a mesma que se expõe graças a um complexo estudo reflexivo, indutivo, dedutivo e aprofundado das informações, com base na revisão bibliográfica e teórica de estudos anteriores. Os resultados obtidos propõem as opções e métodos de ensino implícitos no Sistema Preventivo de Dom Bosco, pesquisas que podem ser aplicadas pelos professores durante a formação dos estudantes universitários.

Palavras-chave: Educomunicação, Sistema Preventivo, Educação, Comunicação, Bons cidadãos.

\section{INTRODUCCIÓN}

Para relacionar y comprender este interesante tema sobre los métodos de enseñanza y comunicación utilizados por San Juan Bosco, conocido como Sistema Preventivo (SPDB), se empezará hablando conceptualmente de la educomunicación desde el punto de vista interdisciplinar y transdisciplinar, es decir una ciencia nueva que une a dos labrantíos de conocimientos que habían estado, por mucho tiempo, separados. Actualmente y gracias a las nuevas Tecnologías de la Información y la Comunicación (TIC) y por "sus condiciones de saber se encuentran estrechamente ligadas al acontecer de la humanidad, en calidad de prácticas constitutivas y privativas, cumpliendo un fundamental papel en nuestras sociedades" (Benítez, 2012, p. 19).

Se puede señalar entonces que la educomunicación o educación en medios, también llamada Media Literacy, es una disciplina que defiende la integración de la enseñanza sobre y con los medios de comunicación en el currículo escolar, según el Center for Media Literacy (Paz, 2008). El objetivo es educar en la recepción crítica de los medios de comunicación de manera que el ciudadano sea consciente de cómo se construyen los mensajes que recibe para tomar decisiones más razonadas, pudiendo participar en la sociedad en la que se desenvuelve de una manera libre y responsable democráticamente hablando.

La educomunicación ubica al hombre en nuevos escenarios socioculturales, con un contexto de cambios permanente que permite formar a la sociedad del conocimiento con diferentes espacios abiertos a la búsqueda y desarrollo de sentidos y de posibilidades; es decir, el hombre aplica la educomunicación como parte de su forma de vida, en una época de creatividad educativa, de innovación con nuevos formatos de trabajo en equipo, de generar alianzas estratégicas, de hacer Coworking y acompañar múltiples caminos de desarrollo; pero también de replantear e ir corrigiendo y formando esa nueva sociedad mediática y tecnologizada, gracias a los cambios sociales y al avance de las TIC.

Se debe asumir entonces la educación como un proceso comunicativo que necesita ser construido, analizado y revisado permanentemente, para que las personas se descubran como productoras de cultura a partir de la apropiación critica de los recursos de la información y de la comunicación social. La educomunicación es relacional e implica la construcción y desarrollo de ecosistemas comunicativos abiertos, colaborativos y 
democráticos, facilitadores del aprendizaje y del pleno ejercicio de ciudadanía para una transformación social. La presente investigación plantea las técnicas, formas y modos, que desde la cátedra universitaria deben implementarse basados en el legado de Don Bosco quien propuso una metodología práctica, la misma que en la actualidad se manifiestan en el hacer, el ser, el conocer y el convivir; las cuales corresponden al trabajo, el sentido, la razón y la afectividad, respectivamente; prácticas y perspectivas de vida, que todo docente debe proporcionar a sus estudiantes universitarios hasta convertirlos en excelentes profesionales y buenos ciudadanos.

El estudio objeto de la presente investigación busca fomentar en la comunidad de la Universidad Politécnica Salesiana (UPS), especialmente en docentes y catedráticos, así como de cualquier otra entidad de educación superior, diseminar las prácticas de enseñanza educomunicativas inmersos en el SPDB aplicando las TIC.

\section{REFERENTES HISTÓRICOS SOBRE LA EDUCOMUNICACIÓN}

La Educomunicación parte de las ideas de la pedagogía critica impulsada por Paulo Freire (1921-1997), quien sustentaba una corriente de pensamiento dialógico y que entendía la educación como una formación orientada a la acción, así como de las corrientes semióticas del estructuralismo formal y de los movimientos constructivistas que abogan por metodologías más abiertas y centradas en el estudiante. Surge en Hispanoamérica a mediados de la década de los sesenta para luego extenderse por España. Entre los teóricos más nombrados están Len Masterman, David Buckingham, Robyn Quin, Kathleen Tyner, Mario Kaplún, Robert Aparici, Ignacio Aguaded, Agustín García Matilla y otros (Paz, 2008).

En América Latina, se formaron grupos de estudios como el Instituto Latinoamericano de la Comunicación Educativa ILCE en México. En España se analizó a la educomunicación a través de la corporación de docentes "Drac Magic" en Cataluña, el SOAP (Servicio de Orientación Para Escolares) en Madrid y en la Universidad Nacional de Educación a Distancia UNED, a partir de los años ochenta (Aparici, 2010).

Celestin Freinet, en Francia por los años 1950 dio paso del análisis de textos a la síntesis o construcción de nuevos elementos textuales. Para Freinet "el lenguaje y la comunicación juegan un papel fundamental en los procesos educativos" (Martinez-Salanova, 2014, Sección: Algo de historia, párrafo 1-2). En Canadá, Herbert Marshall McLuhan (19111980), afirmó que el medio es el mensaje, pues la tecnología modifica a la persona, ya que el medio de comunicación influye, determina y moldea, sistema que denominó "Aldea Global" (Martinez-Salanova, 2014). Paulo Freire, Mario Kaplún (1923-1998) y Lev Vygotsky (1896-1934), como parte de sus investigaciones unieron a la educación con el arte, los medios de comunicación, la cultura con la comunicación popular (MartinezSalanova, 2014).

En 1980 la presencia de Len Masterman, da paso a una pedagogía crítica de la educomunicación quien inicia un variado proceso de interacciones con los medios de comunicación, se trata de una nueva forma de educación para igualar a los nuevos medios interactivos promoviendo el desarrollo de destrezas y de pensamiento crítico en torno a las nuevas tecnologías. Pero es Jesús Martín Barbero quien impulsa el sistema sobre los medios a las mediaciones, con lo que abre las puertas a una nueva mirada de los procesos culturales de investigar a los medios en colaboración con las redes sociales (Martinez- 
Salanova, 2014). Otros investigadores de la educomunicación como Daniel Prieto Castillo de Argentina; Ismar de Oliveira Soares de Brasil; Roberto Aparici, Agustín García Matilla, José Manuel Pérez Tornero, José Ignacio Aguaded, de España; coinciden en señalar que la educomunicación es provocar el interés de las personas para que se descubran como productoras de cultura a partir de la obtención de recursos de información y comunicación social, es decir se trata de "un acto de concienciación, donde todo proceso educativo es un acto comunicativo", Aparici en Martinez-Salanova (2014, párrafos 22 y 25).

\subsection{LA VIDA DE DON BOSCO Y SU ÁMBITO EDUCATIVO Y COMUNICACIONAL}

Juan Melchor Bosco Occhiena (1815-1888) nació en Morialdo, poblado de Castelnuovo de Asti-Italia, el 16 de agosto de 1815, hijo menor del matrimonio compuesto por Margarita Occhiena y Luis Francisco Bosco, "sus hermanos mayores fueron Antonio y José, todos campesinos honrados y trabajadores. Huérfano de padre, quien murió de una terrible pulmonía a los 34 años de edad; sólo después de 24 meses de haber nacido" (Peraza, 2012, p. 18).

A sus 15 años, en 1830 inicia formalmente sus estudios en las escuelas públicas de la ciudad de Chieri, situada al occidente de Monferrato, lugar donde estudió el Seminario, en la catedral Santa María de la Escala hasta 1841. Su primer maestro fue el Padre Eustaquio Velimberti. Su fructífera vida estudiantil transcurrió entre el aprendizaje y la enseñanza, pues "dedicaba parte de su tiempo a ganar dinero transmitiendo los conocimientos adquiridos a otras personas" (Peraza, 2012, p. 65). Ordenado sacerdote en junio de 1841 por el Monseñor Luis Fransoni, mezcla el ambiente de oración, reflexión y estudios, "lo que permitirá a Don Bosco obtener una gran experiencia pastoral entre los muchachos más pobres y abandonados de Turín, que eran guiados por su confesor, el Padre José Cafasso" (Peraza, 2012, p. 141).

Don Bosco aplicó varios procesos educomunicacionales, mezclando los proverbios pastorales de su propia espiritualidad apostólica con los métodos educativos y acciones comunicacionales, dirigida a los muchachos pobres y abandonados, para ello manifiesta la confianza en Dios y plantea que la verdadera fe se la vive en la caridad. -iPorque de las cosas divinas, la mayor es cooperar con el Señor en la salvación del prójimo!- manifiesta Don Bosco. Es decir, aquel que se inventó en la formación del trato educativo con los muchachos pobres y abandonados de las calles; aquel que en los rasgos humanos de los chicos se reflejan con su tónica de espontaneidad, de alegría y de idealismo congénito, propios de su edad, con desalientos, imprudencias, desilusiones, fragilidades, "pero siempre junto a la mano amiga y confidente sincero del maestro, quien acompañe, guie y esté junto siempre a sus estudiantes" (Peraza, 2012, p. 155).

¿Pero cómo deben ser esos nuevos y buenos ciudadanos? Es la pregunta que responde Jorge Benedicto, doctor en sociología y catedrático de la Universidad Nacional de Educación a Distancia UNED:

Esta forma moderna de ver a la ciudadanía tiene una visión liberal y occidental, expresada en el concepto de ciudadanía que construye Marshall sobre una comprensión en torno a principios de igualdad, justicia, derechos y obligaciones, visión tradicional entendida como estática y desligada de la sociedad donde las desigualdades sociales y el divorcio entre el Estado y el pueblo está a la vista actualmente de manera uniforme y sin participación (Salesiana, 2016, p. 26). 
El Padre Geraldo Caliman, doctor de la Universidad Pontificia Salesiana de Roma y profesor de la Universidad Católica de Brasilia, coincide con Benedicto al señalar que la pedagogía social no se da solamente en la escuela, sino también en la familia, en los espacios culturales, en los medios de comunicación, en el día a día de la sociedad, pues a su criterio se trata de una pedagogía eminentemente salesiana ligada a las ciencias de la educación y a las ciencias sociales, orientada a potenciar la sociabilidad, priorizar las relaciones humanas, fortaleciendo el ambiente afectivo que provocan las condiciones de cambio personal; y su característica se basa en ser afectiva, racional, tecnológica y existencial, "es algo así como una obra de arte que da sentido a la vida de nuestros estudiantes universitarios" (Salesiana, 2016, p. 27). Además, que propugne que los medios se conviertan en material de aula para que los estudiantes aprendan a deconstruir y construir mensajes, a fin de comprender los engranajes que sostienen el proceso de producción mediática desde una amplia perspectiva en el ámbito social, estética, política, económica, etc. (Cabrera, 2010). Sin dejar de lado el conocimiento de que nuestras sociedades latinoamericanas se caracterizan por vivir estados de dominación interna y externa, el proyecto educomunicativo debería ser un saber crítico de esta situación ya que los medios generalmente forman parte de las estructuras dominantes (Ogaz, 2002). Postura que se suma a la crítica antropológica de (Geertz, 1996) quien señala que "por todos lados la reivindicación etnográfica del conocimiento está siendo puesta bajo la duda moral, recalificada como una impronta de poder" (p. 131).

\subsection{EL SISTEMA PREVENTIVO Y EL PENSAMIENTO PEDAGÓGICO DE SAN JUAN BOSCO}

Para la profesora Rosa María Torres, la pedagogía salesiana se adelantó crítica y visionariamente a su época y no ha perdido vigencia, sus principios y orientaciones tiene actualmente plena validez, se trata de la importancia otorgada a los jóvenes, la prevención de los problemas, el diálogo, la empatía, el acompañamiento permanente, la organización, la participación y el protagonismo juvenil, "todo ello en un mundo que vive ensañándose con los jóvenes y minando derechos fundamentales como el estudio, el trabajo y la esperanza de un futuro mejor" (Torres, 2013, p. 24).

"Don Bosco les enseñaba a leer y escribir, también el catecismo, buscaba trabajo para los que no tenían empleo, pan para los hambrientos, y zapatos para los que llegaban descalzos" (Sálesman, 2006, p. 11). Sus enseñanzas eran a través del juego y el canto, actividad que duró más de tres años hasta cuando fue nombrado capellán de un colegio de niñas huérfanas y tuvo que cambiarse con toda su "pandilla". Después de ocho meses se trasladó a una capilla abandonada, pues sus muchachos sumaban los 400 jóvenes. "Fue expulsado siete veces de varios sitios donde pernoctó hasta que finalmente alquiló una antigua granja, la que con la ayuda de todos sus chicos logró convertirla en la Capilla de San Francisco de Sales, donde Don Bosco vivió más de 43 años hasta su muerte" (Sálesman, 2006, pp. 12-13).

El método que Don Bosco usaba para educar, lo llamaba Sistema Preventivo, consiste en prevenir o anticipar siempre al joven antes de castigarlo. Si deja de portarse mal ya no se le castiga, tratarlo siempre con la más exquisita bondad. Evitar en lo posible los castigos y no usar nunca palabras duras. Razonarle todo lo que se le ordena y hacer que viva en gracia de Dios por medio de frecuentes confesiones y comunión, asistir a la Santa misa y realizar muchas oraciones breves con una devoción fuerte hacia la 
Santísima Virgen. Este es el sistema con el que Don Bosco cambiaba a los jóvenes en pocos meses, lo que provocaba admiración en sus familias (Sálesman, 2006, p. 123).

El Padre José Bastidas y el licenciado Eduardo Paucar, autores del libro El Sistema Preventivo de Don Bosco, explican: "El concepto de -prevención- manejado por Don Bosco, no es de carácter puramente asistencial y protectivo" (Bastidas y Paucar, 2012). Sin tener que prescindir de dichas situaciones, "será necesario la instrucción y preparación de la persona para evitar su perdición, o sea que lo preventivo es promoción social, es decir una completa capacitación de la persona en todos los ámbitos de vida" (p. 12).

Mientras que el Padre jesuita Alberto Caviglia en Fierro (1950), considera a Don Bosco el "pedagogo genial" por su propia y creativa originalidad para enseñar, pues a su criterio, la pedagogía de Don Bosco no está solo limitada al campo de la formación cristiana, sino que se aplica a la metodología general de enseñanza y particularmente a toda comunicación didáctica; es decir el Santo aplicaba plenamente la Educomunicación en sus jóvenes estudiantes en Italia, como se ha explicado en páginas anteriores. En el libro La Pedagogía Social de Don Bosco, de Rodolfo Fierro Torres (pp. 29-32) coincide con Bastidas al señalar en que consiste el Sistema Preventivo y por qué debe preferirse:

El alumno preventivamente avisado no queda desalentado por la falta cometida, pues el maestro en vez de amenazar con castigarlo deberá de acompañarlo con un aviso amistoso y preventivo, con lo que se gana al estudiante, quien ve en su profesor a un bienhechor que le avisa, que le hace bien, librándole de sinsabores, del castigo y de la deshonra (Fierro, 1950).

El propósito de Don Bosco no solo es la de prevenir o evitar lo negativo o dañino que pueda presentarse en situaciones en las que se desenvuelven los estudiantes para que el joven no sufra en el diario vivir, la idea era hacer que el alumno tome conciencia y ayudándolo pueda defenderse de circunstancias adversas que puedan presentarse en su vida. Para ello Don Bosco propone metas, impulsando procesos de crecimiento, "más aún en aquellos jóvenes involucrados en experiencias delincuenciales, pues para ellos, se requería una atención inmediata y prioritaria por parte del educador" (Bastidas et al., 2012, p. 13).

Se debe preferir y aplicar el SPDB por que dispone y persuade de tal modo al alumno, que el educador podrá en cualquier ocasión hablarle con el lenguaje del amor, la razón y la religión, conquistando su corazón, con lo que el educador podrá ejercer sobre él, gran influencia para corregirlo y aconsejarle, cada vez que sea necesario. Prellazo cita al Padre Giacomo Costamagna, quien fue Obispo de Ecuador, misionero de América y colaborador de Don Bosco y quien recibió una carta del Santo donde puntualmente le señaló: "El Sistema Preventivo sea nuestra característica: Nunca castigos físicos, nunca malas palabras, nunca represiones severas delante del otro. Por lo contario, que en las clases suenen palabras: dulzura, caridad y paciencia. Dar a todos mucha libertad y mucha confianza" (Prellezo, 2004, p. 26).

(Torres, 2013) señala que la investigación neurológica está confirmando lo que siempre se ha conocido; más allá de métodos y contenidos, lo que más importa en el aprendizaje y en la relación pedagógica es el afecto. Cualidades esenciales de todo buen educador deben ser la empatía, vocación de servicios, simpatía, cordialidad, buen humor, actitudes conciliadoras, no violentas, credibilidad, fomentar el juego, el arte, la recreación, la expresión 
corporal, la risa, el humor, el contacto con la naturaleza, etc., "son claves importantes para una pedagogía del afecto, sumados a importantes valores como la colaboración y la solidaridad, el respeto, la honestidad, la austeridad, se aprenden viendo del docente ejercerlos y ejerciéndolos" (pp. 27-28). A lo señalado por Torres, debemos considerar los valores de la pedagogía de Don Bosco que tiene su expresión más característica en la fiesta, y como todos sabemos a los jóvenes les gustan las fiestas, para Don Bosco todo lo que sirve al crecimiento de los jóvenes, está ligada a los objetivos de su pedagogía como parte de la visión optimista de la vida y de la educación de los jóvenes, ya que su principal axioma pedagógico era que todos estén alegres. Torres (2013) cuenta que:

En las fiestas proverbiales del Oratorio entraban todas las riquezas personales y se evocaban y rehacían las tradiciones de la cultura popular. El Juego, el teatro y la música pertenecen al lenguaje pedagógico de la fiesta usado por Don Bosco, pero también la oración y la liturgia (p. 24).

\section{METODOLOGÍA DE INVESTIGACIÓN}

La metodología aplicada fue de enfoque cualitativa, se utiliza para "la recolección y análisis de los datos para afinar las preguntas de investigación o revelar nuevas interrogantes en el proceso de interpretación" (Hernández-Sampieri et al., 2014, p. 7). Y de tipo descriptiva que "busca especificar propiedades y características importantes de cualquier fenómeno que se analice" (p. 92); y explicativa, para especificar las propiedades, características, perfiles de personas o grupos de personas sometidos a un completo análisis, así como las causas y efectos del fenómeno tratado con relación al tema objeto de investigación, la misma que se expone gracias a un complejo estudio reflexivo inductivo, deductivo y de profundización de la recopilación, selección y clasificación de la información, basada en la revisión bibliográfica y teórica procedente de estudios anteriores.

Además, se encuestó a 232 estudiantes de la UPS para medir los niveles de conocimiento sobre el SPDB y su aplicación durante los procesos de aprendizaje; y se trabajó con la herramienta de observación no participante, que "es una técnica útil, especialmente, permite apoyar el mapeo librando al investigador a una descalificación por -incompetencia cultural-. Contar con un registro estructurado sobre ciertos elementos básicos para comprender la realidad humana objeto de análisis" (Quintana, 2006, p. 67).

\section{RESULTADOS DE LA INVESTIGACIÓN}

Teniendo en cuenta la importancia del pensamiento pedagógico y del SPDB en la formación de estudiantes universitarios, se realizó el análisis descriptivo para identificar qué conocían los jóvenes sobre el tema, se realizó una encuesta online a 232 estudiantes de la Universidad Politécnica Salesiana sede Guayaquil, quienes respondieron a 16 ítems del cuestionario de preguntas: (ver descriptores completos en: https://bit.ly/3lub0E9).

Desde la primera hasta la sexta pregunta, la encuesta recogió datos sociodemográficos que indican que de 232 encuestados: 146 son de género masculino y 86 femenino; y sus edades fluctúan entre 18 y 25 años con un nivel de estudios que alcanza a 194 universitarios, 
30 bachilleres, siete con estudios de curato nivel, y uno con estudio secundario. Todos de nacionalidad ecuatoriana y habitantes radicados en la ciudad de Guayaquil. Sobre la pregunta siete, que consultaba si conocen del SPDB, 112 universitarios respondieron no conocer sobre el SPDB, y el mismo número de encuestados dijo que si conocen, mientras que ocho jóvenes no respondieron, tal como se observa en la figura 1.

Figura 1. Sistema Preventivo de Don Bosco

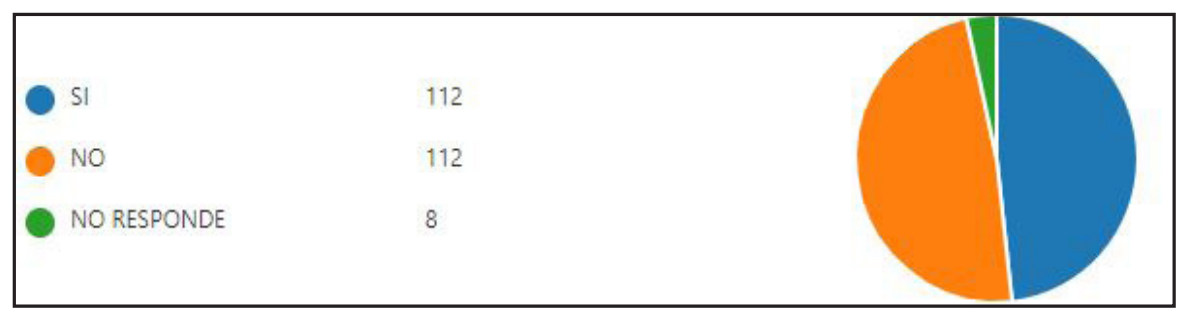

Fuente: Elaboración propia con datos de Google Forms.

Al consultar que tan importante para la educación universitaria es el SPDB, 124 encuestados creen que es muy importante; 45 extremadamente importante; 52 medio importante; y 11 nada importante.

Figura 2. Importancia del SPDB en la educación superior

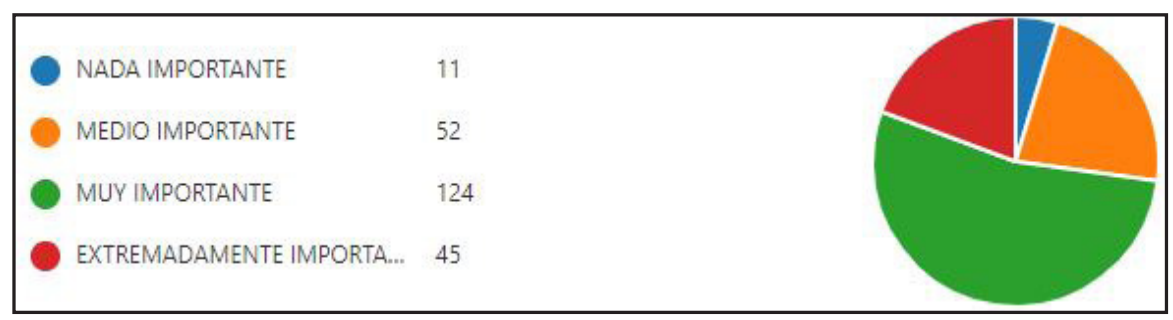

Fuente: Elaboración propia con datos de Google Forms.

De los 232 encuestados, 99 respondieron no conocer algún docente que utilice el SPDB mientras dictan sus clases, 84 si conocer a docentes aplicar el SPDB, y 49 no respondieron; Sin embargo, 160 si están de acuerdo en que todos los docentes universitarios apliquen el SPDB mientras dictan sus clases, 60 jóvenes no respondieron, y 12 no están de acuerdo, como se observa en la Figura 3 y 4. 
Figura 3. Docente que utilizan el SPDB

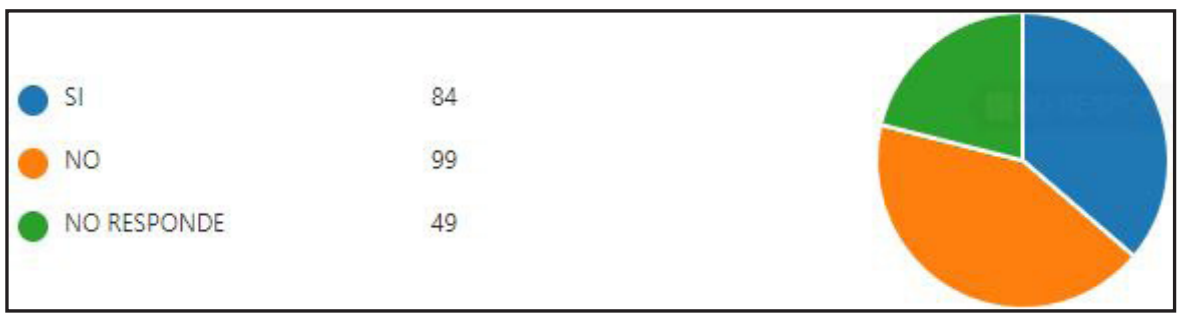

Fuente: Elaboración propia con datos de Google Forms.

Figura 4. Todo docente debe utilizar el SPDB

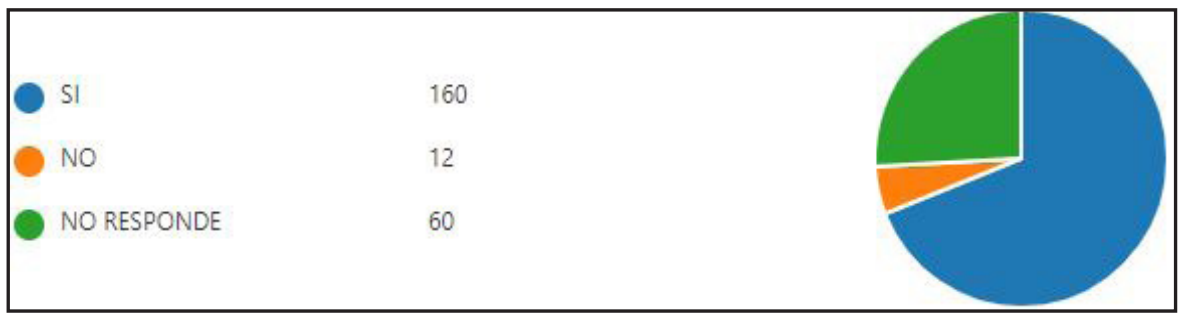

Fuente: Elaboración propia con datos de Google Forms.

La encuesta aplicada sirvió también para que los universitarios encuestados califiquen el sistema educativo aplicado en el proceso de enseñanza aprendizaje al recibir clases: seis lo calificaron como malo, 30 regular, 73 bueno, 84 muy bueno, y 39 excelente.

Figura 5. Calificando el sistema educativo actual

\begin{tabular}{|ll|}
\hline MALO & 6 \\
REgular & 30 \\
BUENo & 73 \\
MUY Bueno & 84 \\
EXCELENTE & 39 \\
\hline
\end{tabular}

Fuente: Elaboración propia con datos de Google Forms.

La pregunta 12 del cuestionario consultó si los docentes deben castigar a los estudiantes que cometen actos de indisciplina; y 149 universitarios respondieron que sí, 50 dijeron no, y 31 no respondieron. 
Figura 6. Castigos para estudiantes indisciplinados

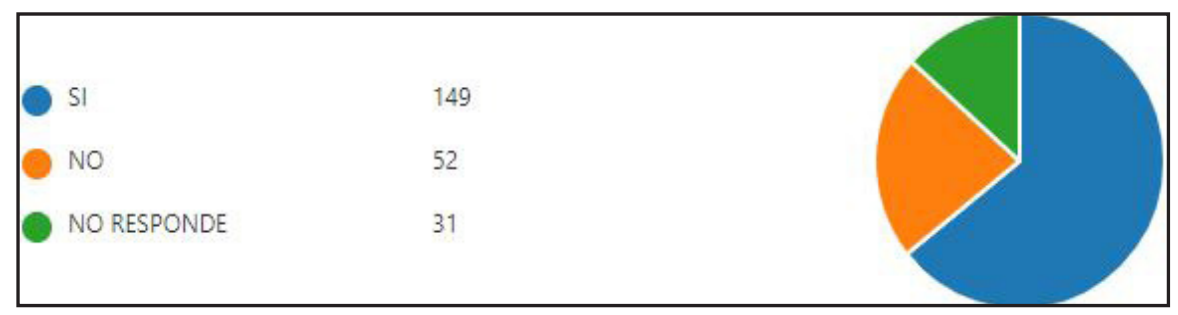

Fuente: Elaboración propia con datos de Google Forms.

Para conocer qué cualidades especiales deberían caracterizar el docente al dictar su clase, se aplicó varias opciones de respuestas, las mismas que son parte de la pedagogía de San Juan Bosco inmersas en el SPDB. 79 encuestados escogieron la "capacidad de reconsiderar" como cualidad especial que siempre deben aplicar los docentes con sus estudiantes; 70 seleccionaron "diálogo fecundo"; 40 escogieron "fuerte personalidad"; 38 "agudeza de percepción"; y apenas cinco encuestados optaron por "ausencia de distancia".

Figura 7. Cualidades especiales de un docente

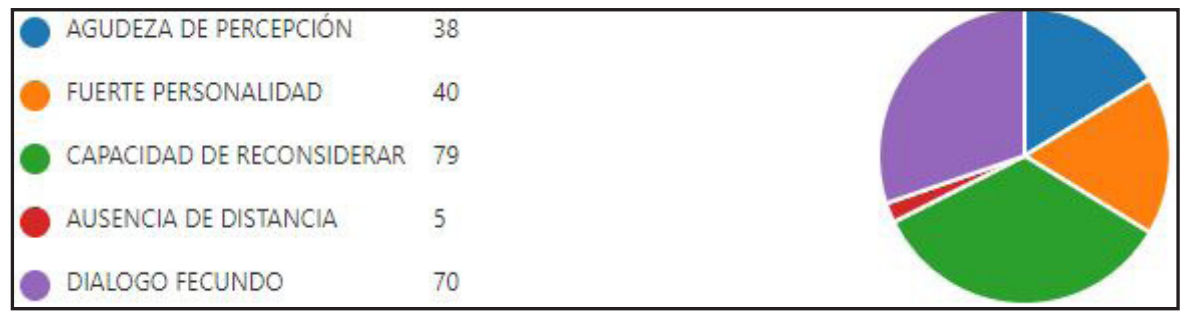

Fuente: Elaboración propia con datos de Google Forms.

Al consultarles a los 232 encuestados ¿si la preparación universitaria recibida sirvió para formarlos como buenos cristianos y honrados ciudadanos? 190 respondieron afirmativamente, 21 dijeron lo contrario; y el mismo número de universitarios no respondieron nada, como lo indica la siguiente figura. 
Figura 8. Universitarios formados como buenos cristianos

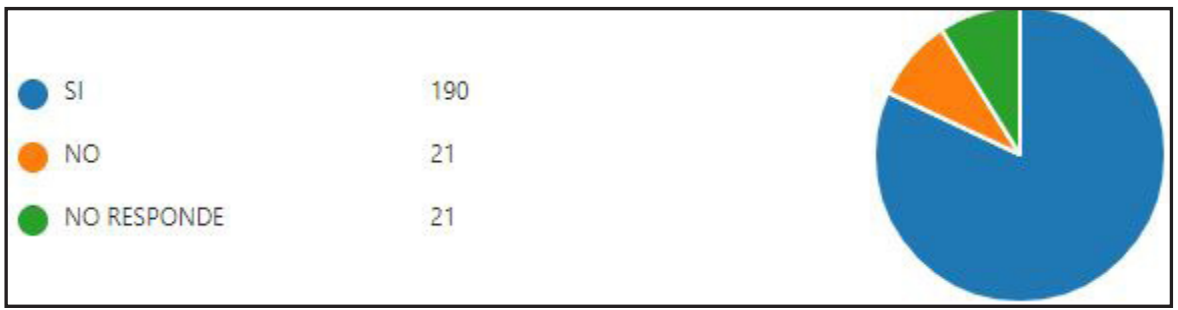

Fuente: Elaboración propia con datos de Google Forms.

De los resultados obtenidos en la pregunta 15 de la encuesta online, 175 universitarios consideran que el proyecto educativo de Don Bosco debe ser aplicado como una alternativa pedagógica en el sistema educativo superior; 14 no están de acuerdo; y 43 no respondieron nada.

Figura 9. Proyecto educativo de Don Bosco: alternativa pedagógica

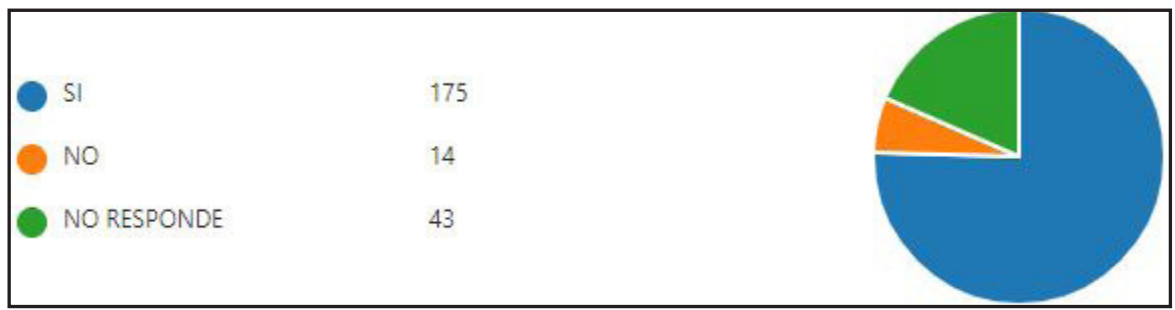

Fuente: Elaboración propia con datos de Google Forms.

La última pregunta del cuestionario consultaba: ¿cómo le gustaría que enseñen los docentes mientras dictan clases? Y la respuesta generalizada y más relevante de los 232 encuestados coinciden en pedir a sus docentes hacer el uso del diálogo con los alumnos para una mejor convivencia, de forma respetuosa, didacta, interactiva, creativa, participativa, entretenida; un poco más de tolerancia, pero estricto; que enseñen con el corazón y la sonrisa, con respeto, paciencia y disciplina, que sean dinámicos en sus clases, con carisma y creatividad, con amor, pasión y empatía.

\section{DISCUSIÓN Y CONCLUSIONES}

La conclusión engloba destacando que la educomunicación de San Juan Bosco, aplicada desde el SPDB, como modelo pedagógico de enseñanza aprendizaje, se presenta como el mejor proceso que todo catedrático debería aplicar a sus estudiantes universitarios hasta convertirlos en buenos ciudadanos, se trata del planteamiento implícito en la presente investigación la misma que describe las formas y aplicaciones de cómo construir a jóvenes 
estudiantes en profesionales responsable, honrados y trabajadores, comprometidos con la familia y la sociedad toda, a diferencia de otras metodologías de enseñanzas, como lo plantea el análisis comparativo entre los modelos educativos y de los medios de comunicación definidos como bancario, conductista o participativo, realizado por el pedagogo Mario Kaplún, quien dedicó su vida a la comunicación educativa y a formar a las comunidades en los procesos participativos de producción de comunicación como una forma de acción y construcción ciudadana (Escalante, 2012).

La presente investigación concluye además en señalar que desde el SPDB, la educomunicación de San Juan Bosco y su metodología pedagógica aplicada para comunicar educando, tiene sus bases en la "sonrisa", es decir utilizando los sentimientos de amor y afecto que el docente deberá aplicar mientras enseña a sus estudiantes; El correcto uso de la "palabra", el conocimiento y la producción de la ciencia a través de la razón, es decir aplicando el razonamiento lógico y responsable; y finalmente haciendo uso del "testimonio" desde la religión, como una forma de vida de los estudiantes, sostenida desde el Creador y en solidaridad con el prójimo. Adicionalmente la metodología de enseñanza de San Juan Bosco promueve los espacios para el encuentro entre estudiantes y docentes fortaleciendo la comunicación asertiva, lo que dará como resultado la formación de buenos cristianos y honrados ciudadanos.

La educomunicación como metodología es un proceso de aprendizaje autónomo para toda la vida y su objetivo está en que los estudiantes apliquen el pensamiento crítico a nuevas situaciones que se presenten en la sociedad, produciendo y desarrollando ecosistemas educomunicativos abiertos, dialógicos y participativos. Aunque el presente trabajo de investigación no es el primero que hace referencia a los pensamientos y métodos pedagógicos de San Juan Bosco en la construcción de buenos ciudadanos; el estudio propone futuras líneas de investigación que fomenten el uso y la práctica del SPDB, el mismo que desde la praxis, como está concebido pedagógicamente ha dado excelentes resultados en la formación académica y humanística de generaciones de estudiantes en todo el mundo y de manera puntual entre la comunidad Salesiana en Ecuador.

\section{REFERENCIAS BIBLIOGRÁFICAS}

Aparici, R. (2010). Educomunicación: más allá del 2.0. Barcelona: Gedisa.

Bastidas, J. \& Paucar, E. (2012). El Sistema Preventivo de Don Bosco. Quito: Imprenta del Colegio Técnico Don Bosco.

Benítez, C. (01 de agosto de 2012). Repositorio Institucional Universidad Politécnica Salesiana. Obtenido de Educomunicación una estrategia para el cambio. Caso: Colegio Intercultural Bilingüe Jatari de la Comunidad de Sarahuasi. https://bit.ly/34iewdD

Cabrera,E.(2010). UniversidaddelAzuay. ObtenidodeEficiencias de programas educomunicacionales. https://bit.ly/3cQWLpH

Escalante, M. (20 de julio de 2012). Portafolio Teoría de la Comunicación. Obtenido de Teoricos Latinoamericanos y Venezolanos de la Comunicación. https://bit.ly/34nk9sy

Fierro, R. (1950). La pedagogía social de Don Bosco. Buenos Aires: Talleres Gráficos Pedemonte Hnos y Stotland.

Geertz, C. (1996). Tras los Hechos. Dos paises, cuatro décadas y un antropólogo. Barcelona: Paidós. Hernández-Sampieri, R., Fernandez, C. \& Baptista, P. (2014). Metodología de la investigación. (6ta. ed.). México D. F.: McGRAW-HILL / Interamericana Editores, S. A. 
Martinez-Salanova, E. (21 de mayo de 2014). Educomunicación. Obtenido de Volver a Educación y Didáctica. https://bit.ly/34xwmem

Ogaz, L. (02 de julio de 2002). Dialéctica Educomunicación. Obtenido de Repositorio Institucional de la Universidad Politécnica Salesiana. https://dspace.ups.edu.ec/handle/123456789/10391

Paz, S. (12 de Mayo de 2008). Centro Regional de Innovación y Formación. Obtenido de Departamento TIC del CRIF Las Acacias. https://bit.ly/3in8ygz

Peraza, F. (2012). Memorias del Oratorio de San Francisco de Sales por San Juan Bosco. Cuenca Ecuador: Centro Gráfico Salesiano.

Prellezo, J. (2004). El Sistema Preventivo en la educación. Madrid: Editorial Biblioteca Nueva.

Quintana, A. (2006). Metodología de investigación científica cualitativa. En A. Quintana \& W. Montgomery, Pisología tópicos de actualidad (pp. 47-84). Lima: UNMSQ. Obtenido de https:// bit.ly/3cVS2TB

Salesiana, U. P. (2016). Congreso Buenos Ciudadanos y Sistema Preventivo: Aportes para buenas prácticas. Quito: Imprenta Don Bosco.

Sálesman, E. (2006). Las aventuras de Don Bosco. Cuenca, Ecuador: Don Bosco Librería LNS.

Torres, R. (2013). La pedagogía salesiana y el actual contexto internacional. En A. Yala, Congreso Nacional Pedagogía de Don Bosco: Reflexiones, experiencias y desafíos. Memoria Académica. (pp. 23-31). Cuenca: Abya Yala. 
